\title{
MULTIPLE NEURAL NETWORKS WITH ENSEMBLE METHOD FOR AUDIO TAGGING WITH NOISY LABELS AND MINIMAL SUPERVISION
}

\author{
Kexin $\mathrm{He}^{*}$, Yuhan Shen ${ }^{*}$, Wei-Qiang Zhang ${ }^{\dagger}$ \\ Beijing National Research Center for Information Science and Technology \\ Department of Electronic Engineering, Tsinghua University, Bejing 100084, China \\ hekexinchn@163.com, yhshen@hotmail.com, wqzhang@tsinghua.edu.cn
}

\begin{abstract}
In this paper, we describe our system for the Task 2 of Detection and Classification of Acoustic Scenes and Events (DCASE) 2019 Challenge: Audio tagging with noisy labels and minimal supervision. This task provides a small amount of verified data (curated data) and a larger quantity of unverified data (noisy data) as training data. Each audio clip contains one or more sound events, so it can be considered as a multi-label audio classification task. To tackle this problem, we mainly use four strategies. The first is a sigmoid-softmax activation to deal with so-called sparse multi-label classification. The second is a staged training strategy to learn from noisy data. The third is a post-processing method that normalizes output scores for each sound class. The last is an ensemble method that averages models learned with multiple neural networks and various acoustic features. All of the above strategies contribute to our system significantly. Our final system achieved labelweighted label-ranking average precision (lwlrap) scores of 0.758 on the private test dataset and 0.742 on the public test dataset, winning the 2nd place in DCASE 2019 Challenge Task 2.
\end{abstract}

Index Terms - Audio tagging, noisy label, model ensemble, DCASE

\section{INTRODUCTION}

The Detection and Classification of Acoustic Scenes and Events (DCASE) Challenge is gaining increasing interests among researchers with academic and industrial backgrounds. DCASE 2019 is the fifth edition of this challenge and has been held to support the development of computational scene and event analysis methods. This paper describes the methods we adopted to participate in the task 2 of DCASE 2019 Challenge.

The second task of this year's challenge is Audio tagging with noisy labels and minimal supervision [1]. It provides public dataset [2] with baseline and aims to develop competitive audio classification systems using a small set of manually-labeled data and a larger set of noisy-labeled data.

State-of-the-art methods are based on deep neural networks, including Convolutional Neural Network (CNN), Recurrent Neural Network (RNN) and Convolutional Recurrent Neural Network (CRNN). We follow this trend and use two types of neural network architectures: a CRNN and a variant of CNN (DenseNet).

\footnotetext{
* The first two authors contributed equally.

${ }^{\dagger}$ This work was supported by the National Natural Science Foundation of China under Grant No. U1836219. The corresponding author is Wei-Qiang Zhang.
}

Data augmentation has been widely utilized in recent DCASE Challenges. Mixup [3] strategy was adopted by top teams [4, 5] in DCASE 2018 Challenge. Besides, a new augmentation method named SpecAugment [6] has been proposed recently. In our work, we used the combination of both methods.

Although this is a multi-label classification problem, most audio clips contain only one sound event. For this reason, we call it a "sparse multi-label classification" problem, and propose a sigmoid-softmax activation structure to deal with this problem.

In this task, how to use noisy data is the key to achieving competitive performance. We designed a staged training strategy to select the most convincing samples from noisy data and train our model using both verified data and convincing unverified data. This new training strategy will be illustrated in the following sections.

Additionally, we did some explorations about post-processing and found an effective way of score normalization.

The rest of this paper is organized as follows: we describe our methods in detail in Section 2; we present our experiments and results in Section 3; finally we conclude our work in Section 4.

\section{METHODS}

\subsection{Feature Extraction}

We used two types of acoustic features, including log-mel energies and perceptual Constant-Q transform (p-CQT). And we also used different parameters, such as frame length, hop length, frequency range, mel bins. As shown in Table 1, we used three feature configurations in total. All features are extracted using librosa [7].

Table 1: Configurations of acoustic features

\begin{tabular}{cccc}
\hline & Type A & Type B & Type C \\
\hline Feature & log-mel & log-mel & CQT \\
Window length & 1764 & 2048 & - \\
Hop length & 882 & 511 & 512 \\
Low Frequency & $0 \mathrm{~Hz}$ & $0 \mathrm{~Hz}$ & $55 \mathrm{~Hz}$ \\
High Frequency & $22050 \mathrm{~Hz}$ & $16000 \mathrm{~Hz}$ & - \\
Feature dim & 80 & 128 & 128 \\
bins per octave & - & - & 16 \\
\hline
\end{tabular}

\subsection{Data Preprocessing}

Raw feature data needs further preprocessing before being input to neural networks. The data preprocessing procedures used in our work include sound activity detection (SAD) and data padding. 

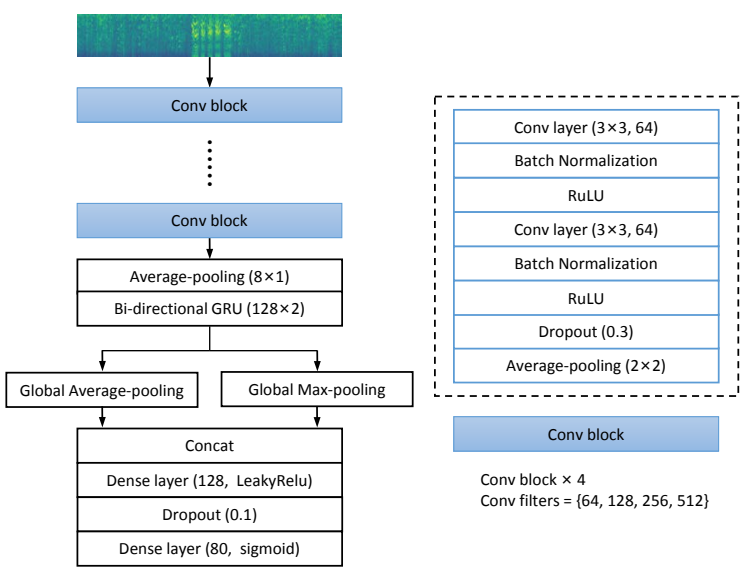

Figure 1: The architecture of CRNN. It consists of 4 convolutional blocks, a bi-GRU and two dense layers. Each convolutional block contains 2 convolutional layers. After bi-GRU, global average pooling and global max pooling operations are applied to aggregate temporal information, and the results are concatenated together before being input to dense layers.

SAD has shown powerful performance in previous work [5]. We mainly used two kinds of SAD methods: 1) We ignore the silent frames at the beginning and end of each audio. 2) We ignore the silent frames through the whole audio.

To deal with the variable lengths of acoustic features, we set a maximum padding length. All features shorter than the padding length will be repeated to the padding length. And longer features will be downsampled to align with the padding length. In our work, the padding length is set 2000. During training, we randomly select continuous 512 frames to feed into the neural network. For test, the whole 2000 frames are used to get predictions.

\subsection{Data Augmentation}

As mentioned above, we combined mixup [3] and SpecAugment [6] for data augmentation.

In mixup, we randomly select a pair of samples from training data. Let $x_{1}, x_{2}$ be the features, and $y_{1}, y_{2}$ be the one-hot labels respectively, the data is mixed as follows:

$$
\begin{aligned}
& x=\lambda x_{1}+(1-\lambda) x_{2} \\
& y=\lambda y_{1}+(1-\lambda) y_{2}
\end{aligned}
$$

where the parameter $\lambda$ is a random variable with Beta distribution $\mathrm{B}(0.4,0.4)$.

SpecAugment is implemented by time warping, frequency masking and time masking. Detail is available in [6].

\subsection{Neural Network}

\subsubsection{CRNN architecture}

The architecture of CRNN is illustrated in Figure 1. It begins with four convolutional blocks. Each block contains two convolutional layers, followed by batch normalization [8], ReLU, dropout [9] and average pooling. Next, an average pooling is adopted on frequency axis to squeeze the frequency dimension to 1 . And a bi-directional
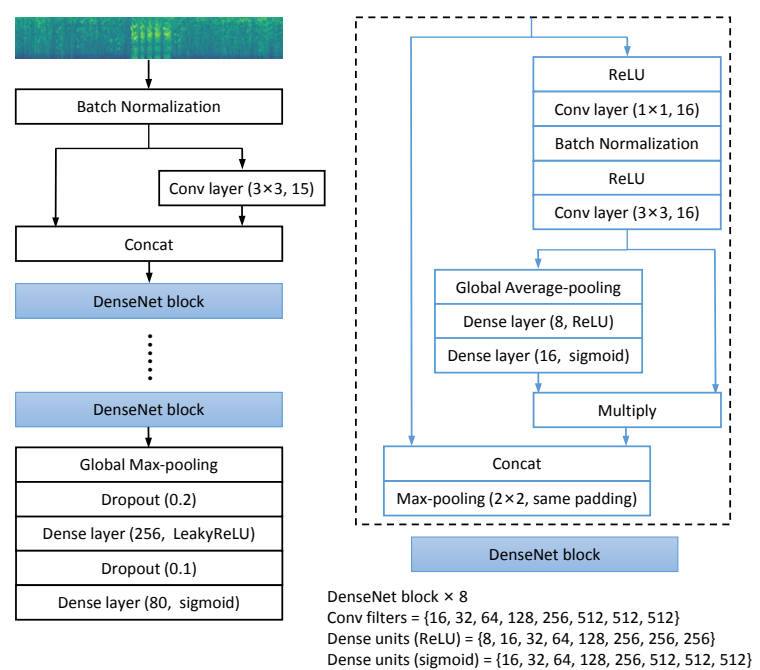

Figure 2: The architecture of DenseNet. Batch normalization is applied to the input acoustic feature, followed by a convolutional layer. The input and output of this convolutional layer are concatenated along channels, followed by 8 DenseNet blocks. Then, global max pooling is applied, and 2 dense layers are utilized to output final predictions. The configuration of DenseNet block is illustrated in the dotted box.

Table 2: The number of positive labels in training dataset

\begin{tabular}{ccc}
\hline \#positive labels & train curated & train noisy \\
\hline 1 & 4269 & 16566 \\
2 & 627 & 2558 \\
3 & 69 & 504 \\
4 & 4 & 141 \\
5 & 0 & 38 \\
6 & 1 & 4 \\
7 & 0 & 4 \\
\hline average \#positive labels & 1.157 & 1.211 \\
percentage of single label & $85.9 \%$ & $83.6 \%$ \\
\hline
\end{tabular}

gated recurrent unit (Bi-GRU) is used to capture temporal context. Then, global max pooling and global average pooling are used on time axis to maintain various information and concatenated together. Finally, two dense layers are applied to output prediction scores for each class.

\subsubsection{DenseNet architecture}

The architecture of DenseNet is shown in Figure 2. Our module is similar to that in [4]. In this module, the feature maps of previous layers can propagate to later layers, which can effectively alleviate the vanishing-gradient problem and encourage feature reuse. In each DenseNet block, we use Squeeze-andExcitation Network [10], which can adaptively recalibrate channelwise feature responses by explicitly modelling interdependencies between channels.

\subsubsection{Choice of final activation}

Since this is a multi-label and multi-class classification task, sigmoid is naturally the primary choice of the activation in final 


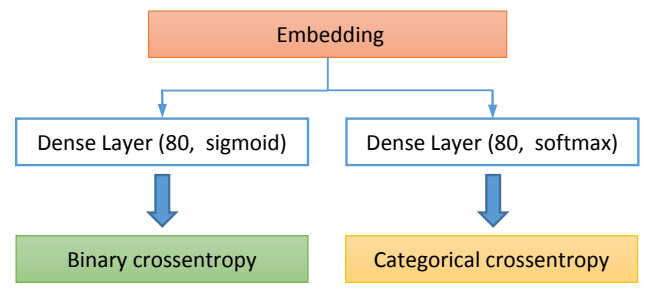

Figure 3: Combination of sigmoid and softmax activation.

layer. However, as shown in Table 2, the average number of positive labels in training dataset is very close to 1 , and single-label data takes up the majority. So we call this task a "sparse multi-label classification" problem. In this condition, softmax is also a good option.

In order to combine the advantages of both sigmoid and softmax, we design a new structure named sigmoid-softmax activation. In this structure, the output embedding before the final layer will pass through two dense layers. As shown in Figure 3, one dense layer with sigmoid activation will be optimized with binary crossentropy loss, and the other dense layer with softmax activation will be optimized with categorical crossentropy loss. The outputs of both dense layers are ensembled to get final prediction.

\subsection{Staged Training Strategy for Noisy Data}

In this task, only a small amount of data is manually labeled, and a large quantity of data contains noisy label. Since the noisy data is not verified to have groundtruth label, we attempt to use only the most convincing noisy data. Inspired by the batch-wise loss masking in [4], we propose a staged training strategy to learn from noisy data.

To make it specific, we firstly use the verified data to train our system for several epochs. Then, we use both the verified and unverified data. However, in order to use only the most convincing noisy data, we adopt a loss masking similar to the work in [4]. The difference is that we ignore the noisy samples with the top $k$ loss in a batch rather than set a threshold value and ignore samples with higher loss. Finally, after training for more epochs, we abandon the noisy data and finetune our model with only the verified data. Our staged training strategy has made huge improvements according to our experiments.

\subsection{Score Normalization}

For inference, we use score normalization strategy for further improvements. Let $x_{i, j}$ be the prediction score for the $i$-th class in the $j$-th sample. We normalize the prediction scores for each class. The normalization procedure goes as follows:

$$
\begin{gathered}
\bar{x}_{i}=\frac{\sum_{j=1}^{N} x_{i, j}}{N}, \\
\hat{x}_{i, j}=\frac{x_{i, j}-\bar{x}_{i}}{\sqrt{\frac{1}{N} \sum_{j=1}^{N}\left(x_{i, j}-\bar{x}_{i}\right)^{2}+\varepsilon}} \\
\widetilde{x}_{i, j}=\frac{\hat{x}_{i, j}-\min _{j} \hat{x}_{i, j}}{\max _{j} \hat{x}_{i, j}-\min _{j} \hat{x}_{i, j}+\varepsilon}
\end{gathered}
$$

where $N$ is the total number of samples in evaluation dataset, $\varepsilon$ is a sufficiently small value to avoid division by zero. For each class in evaluation dataset, we normalize the prediction scores to zero mean and unit variance. Then, we set min and max zoom to keep the scores between 0 and 1 . According to experimental results, score normalization can raise the evaluation metric by approximately 0.002 on average in cross-validation and 0.007 in private test data.

\section{EXPERIMENTS}

\subsection{Experiment Setup}

Adam optimizer [11] is used for gradient based optimization. The learning rate is 0.001 and batch size is 64 . We split our training dataset into four folds. Then we train four models using any three folds for training and the other fold for validation.

As for the staged training, we design a data generator to generate different proportions of training data during different stages. In the first stage, all data comes from curated dataset. In the second stage, the proportion of curated dataset is equal to noisy dataset. In the third stage, only curated dataset is used. In the second stage, the top $k$ samples with the highest loss on noisy dataset would be masked. In our experiments, $k$ is 10 .

For CRNN architecture, the first stage runs for $8 \mathrm{k}$ iterations, the second stage runs for $12 \mathrm{k}$ iterations, and the third stage runs for $3 \mathrm{k}$ iterations. For DenseNet architecture, the first stage runs for $5 \mathrm{k}$ iterations, the second stage runs for $8 \mathrm{k}$ iterations, and the third stage runs for $2 \mathrm{k}$ iterations. The models with the best validation performance on each fold are selected.

\subsection{Evaluation Metric}

The primary competition metric is label-weighted label-ranking average precision (lwlrap). This measures the average precision of retrieving a ranked list of relevant labels for each test clip (i.e., the system ranks all the available labels, then the precisions of the ranked lists down to each true label are averaged). LRAP is calculated as follows, and lwlrap is the macro-average of per-class LRAP. [12]

$$
\operatorname{LRAP}(y, \hat{f})=\frac{1}{n_{\text {samples }}} \sum_{i=0}^{n_{\text {samples }}-1} \frac{1}{\left\|y_{i}\right\|_{0}} \sum_{j: y_{i j}=1} \frac{\left|\mathcal{L}_{i j}\right|}{\operatorname{rank}_{i j}}
$$

where $\mathcal{L}_{i j}=\left\{k: y_{i k}=1, \hat{f}_{i k} \geq \hat{f}_{i j}\right\}, \quad \operatorname{rank}_{i j}=$ $\left|\left\{k: \hat{f}_{i k} \geq \hat{f}_{i j}\right\}\right|,|\cdot|$ computes the cardinality of the set, and $\|\cdot\|_{0}$ computes the number of nonzero elements in a vector.

\subsection{Model Ensemble and Submissions}

Model ensemble is successful in boosting the system's performance. We ensemble our models using geometric average as follows:

$$
y_{\text {ensemble }}=\exp \frac{1}{N} \sum_{n} w_{n} \log y_{n}
$$

where $N$ is the number of subsystems, $y_{n}$ is the output score of each subsystem, and $w_{n}$ is the weight coefficient for each subsystem.

We submitted two prediction results using different weights: 
Table 3: Lwlrap scores of multiple configurations, on both cross-fold validation and private evaluation dataset. The score on cross-fold validation dataset are the average of scores on four folds. "sig-soft" is the abbreviation of sigmoid-softmax activation structure.

\begin{tabular}{c|ccc|ccc}
\hline & \multicolumn{3}{|c|}{ Cross-fold Validation } & \multicolumn{3}{c}{ Private Evaluation } \\
\cline { 2 - 7 } & Type A & Type B & Type C & Type A & Type B & Type C \\
\hline CRNN sigmoid & 0.8512 & 0.8547 & 0.8413 & 0.7253 & 0.7290 & 0.7140 \\
CRNN softmax & 0.8437 & 0.8479 & 0.8362 & 0.7208 & 0.7176 & 0.7094 \\
CRNN sig-soft & $\mathbf{0 . 8 5 6 1}$ & $\mathbf{0 . 8 6 1 3}$ & $\mathbf{0 . 8 5 0 2}$ & $\mathbf{0 . 7 3 8 8}$ & $\mathbf{0 . 7 3 3 4}$ & $\mathbf{0 . 7 2 0 3}$ \\
DenseNet sigmoid & 0.8219 & 0.8357 & 0.8321 & 0.7053 & 0.7017 & 0.6923 \\
DenseNet softmax & 0.8143 & 0.8235 & 0.8249 & 0.7014 & 0.7043 & 0.6837 \\
DenseNet sig-soft & 0.8246 & 0.8378 & 0.8412 & 0.7146 & 0.7141 & 0.7074 \\
\hline
\end{tabular}

Table 4: The performance of systems using curated data only and systems using both curated and noisy data. We use CRNN architecture with three different activation functions on both cross-fold validation and private evaluation dataset. The relative improvement of adding noisy data using staged training strategy is shown in the parenthesis. Type A feature is used in experiments.

\begin{tabular}{c|c|cc}
\hline \multicolumn{2}{l|}{} & Curated data & Curated and noisy data \\
\hline \multirow{2}{*}{ Cross-fold } & sigmoid & 0.8417 & $0.8512(1.13 \% \uparrow)$ \\
Validation & softmax & 0.8278 & $0.8437(1.92 \% \uparrow)$ \\
& sig-soft & 0.8376 & $0.8561(2.21 \% \uparrow)$ \\
\hline \multirow{2}{*}{ Private } & sigmoid & 0.7155 & $0.7253(1.37 \% \uparrow)$ \\
Evaluation & softmax & 0.7142 & $0.7208(0.92 \% \uparrow)$ \\
& sig-soft & 0.7207 & $0.7388(2.51 \% \uparrow)$ \\
\hline
\end{tabular}

1) Zhang_THU_task2_1.output.csv: achieved our highest lwlrap score of 0.742 on public leaderboard in Kaggle.

2) Zhang_THU_task2_2.output.csv: achieved our highest local lwlrap scores in each cross-fold validation, with a lwlrap score of 0.739 on public leaderboard in Kaggle.

\subsection{Experimental Results}

In order to investigate more about proposed methods, we conducted further experiments on private evaluation dataset after submitting to DCASE Challenge. Our experiments were conducted on two neural network architectures (CRNN and DenseNet), three activation functions (sigmoid, softmax, and sigmoid-softmax activation structure), and three acoustic features (Type A, B, C as mentioned in subsection 2.1).

The lwlrap scores of multiple configurations, on both cross-fold validation and private evaluation dataset, are shown in Table 3. The score on cross-fold validation dataset is the average of scores on four folds. As shown in Table 3, CRNN architecture with sigmoidsoftmax activation structure can achieve the best performance in all types of features on both validation and evaluation dataset. Besides, sigmoid-softmax activation structure can outperform single sigmoid or softmax activation in all feature types and neural networks. We can draw a conclusion that proposed sigmoidsoftmax can demonstrate remarkable performance in "sparse multilabel classification" problems.

To examine the performance of proposed staged training strategy, we also conducted some comparative experiments using curated data only. We compare the performance of systems using curated data only and systems using both curated and noisy data in
Table 5: Comparison of several systems, on both public leaderboard and private leaderboard.

\begin{tabular}{c|cc}
\hline & $\begin{array}{c}\text { Lwlrap } \\
\text { (public LB) }\end{array}$ & $\begin{array}{c}\text { Lwlrap } \\
\text { (private LB) }\end{array}$ \\
\hline Ensemble-1 & $\mathbf{0 . 7 4 2 3}$ & 0.7575 \\
Ensemble-2 & 0.7392 & $\mathbf{0 . 7 5 7 7}$ \\
Ensemble-3 & $* * *$ & 0.7508 \\
Ensemble-4 & $* * *$ & 0.7500 \\
\hline OUmed & $\mathbf{0 . 7 4 7 4}$ & $\mathbf{0 . 7 5 7 9}$ \\
Ebbers & 0.7305 & 0.7552 \\
\hline
\end{tabular}

Table 4. We use Type A feature as acoustic feature and CRNN as classifier. Three types of activations are applied to verify the effects of staged training strategy. The results show that proposed method can make good use of noisy data to enhance the classification and generalization capability of our models.

Furthermore, we compare the following systems in Table 5:

- Ensemble-1: the architecture generating aforementioned Zhang_THU_task2_1.output.csv.

- Ensemble-2: the architecture generating aforementioned Zhang_THU_task2_2.output.csv.

- Ensemble-3: the same architecture with Ensemble-1, but without score normalization processing.

- Ensemble-4: the same architecture with Ensemble-2, but without score normalization processing.

- OUmed: the 1st place in DCASE Challenge. [13]

- Ebbers: the 3rd place in DCASE Challenge. [14]

It can be concluded that proposed score normalization strategy can increase lwlrap score by approximately 0.007 . Compared with other teams, our system is also very competitive.

\section{CONCLUSION}

In this paper, we describe our methods and techniques used in the task 2 of DCASE 2019 Challenge. We adopted mixup and SpecAugment for data augmentation and applied two types of deep learning model including CRNN and DenseNet. Besides, a staged training strategy is applied to learn from both curated and noisy data and a sigmoid-softmax activation structure is proposed to solve sparse multi-label classification problems. Using model ensemble and score normalization strategies, our final system ranked the 2 nd place in DCASE 2019 Challenge. 


\section{REFERENCES}

[1] E. Fonseca, M. Plakal, F. Font, D. P. Ellis, and X. Serra, "Audio tagging with noisy labels and minimal supervision," arXiv preprint arXiv:1906.02975, 2019.

[2] E. Fonseca, J. Pons Puig, X. Favory, F. Font Corbera, D. Bogdanov, A. Ferraro, S. Oramas, A. Porter, and X. Serra, "Freesound datasets: a platform for the creation of open audio datasets," in Proceedings of the 18th ISMIR Conference; 2017 oct 23-27; Suzhou, China.[Canada]: International Society for Music Information Retrieval; 2017. p. 486-93. International Society for Music Information Retrieval (ISMIR), 2017.

[3] H. Zhang, M. Cisse, Y. N. Dauphin, and D. Lopez-Paz, "mixup: Beyond empirical risk minimization," arXiv preprint arXiv:1710.09412, 2017.

[4] I.-Y. Jeong and H. Lim, "Audio tagging system for DCASE 2018: focusing on label noise, data augmentation and its efficient learning," DCASE2018 Challenge, Tech. Rep., 2018.

[5] T. Iqbal, Q. Kong, M. D. Plumbley, and W. Wang, "Stacked convolutional neural networks for general-purpose audio tagging," Tech. Rep., DCASE Challenge, 2018.

[6] D. S. Park, W. Chan, Y. Zhang, C.-C. Chiu, B. Zoph, E. D. Cubuk, and Q. V. Le, "Specaugment: A simple data augmentation method for automatic speech recognition," arXiv preprint arXiv:1904.08779, 2019.

[7] B. McFee, C. Raffel, D. Liang, D. P. Ellis, M. McVicar, E. Battenberg, and O. Nieto, "librosa: Audio and music signal analysis in python," in Proceedings of the 14th python in science conference, 2015, pp. 18-25.

[8] S. Ioffe and C. Szegedy, "Batch normalization: Accelerating deep network training by reducing internal covariate shift," in Proceedings of The 32nd International Conference on Machine Learning, 2015, pp. 448-456.

[9] N. Srivastava, G. E. Hinton, A. Krizhevsky, I. Sutskever, and R. Salakhutdinov, "Dropout: a simple way to prevent neural networks from overfitting," Journal of Machine Learning Research, vol. 15, no. 1, pp. 1929-1958, 2014.

[10] J. Hu, L. Shen, and G. Sun, "Squeeze-and-excitation networks," in Proceedings of the IEEE conference on computer vision and pattern recognition, 2018, pp. 7132 7141.

[11] D. P. Kingma and J. Ba, "Adam: A method for stochastic optimization," arXiv preprint arXiv:1412.6980, 2014.

[12] https://www.kaggle.com/c/freesound-audio-tagging-2019/ overview/evaluation.

[13] O. Akiyama and J. Sato, "Multitask learning and semisupervised learning with noisy data for audio tagging," DCASE2019 Challenge, Tech. Rep., 2019.

[14] J. Ebbers and R. Haeb-Umbach, "Convolutional recurrent neural network and data augmentation for audio tagging with noisy labels and minimal supervision," DCASE2019 Challenge, Tech. Rep., 2019. 